\section{Lymph node co-infection of Mycobacterium avium complex and cytomegalovirus in an acquired immunodeficiency syndrome patient}

\author{
Arya Hedjazi, Marzieh Hosseini, \\ Amin Hoseinzadeh \\ Legal Medicine Research Center, Legal \\ Medicine Organization, Tehran, Iran
}

\begin{abstract}
Acquired immunodeficiency syndrome patients are known to have an increased tendency for developing opportunistic infections. However, there are no reports of simultaneous lymph node involvement of cytomegalovirus and Mycobacterium avium complex in a human immunodeficiency virus-positive patient. We report a 31-year-old man who presented with acute abdominal pain and tenderness and weight loss. He died a few hours after admission. Autopsy studies showed coinfection of cytomegalovirus, Mycobacterium avium complex and human immunodeficiency virus. Our case emphasizes the need to be careful in evaluating opportunistic infections in severely immunodepressed acquired immunodeficiency syndrome patients. This case report is the first manifestation of acquired immunodeficiency syndrome in this patient.
\end{abstract}

\section{Introduction}

Human Immunodeficiency Virus (HIV) infection as one of the most significant pandemics in recorded history is a major health concern globally. ${ }^{1}$ Worldwide, more than 33 million people are infected with HIV. ${ }^{2}$ Opportunistic infections are a leading cause of mortality and morbidity in patients living with acquired immunodeficiency syndrome (AIDS). ${ }^{3} \quad$ Disseminated Mycobacterium avium Complex (MAC) infection is an opportunistic infection that was recognized as an end stage complication of AIDS 20 years ago. ${ }^{4}$ There has been increasing interest in disseminated MAC and other nontuberculous mycobacterial infections as a result of the HIV epidemic. ${ }^{5}$

Cytomegalovirus (CMV) is one of the most frequently disseminated opportunistic infections seen with AIDS. ${ }^{6} \mathrm{CMV}$ is frequently diagnosed in HIV patients and an association of CMV and MAC has been reported in cutaneous lesions of AIDS patients. $^{7}$ However, concurrent lymph node involvement of CMV and mycobacteria has not been previously reported.
We report here the discovery of three infections or coinfections at the same time in a young male patient who was hospitalized for abdominal pain. This case report is the first manifestation of AIDS in this patient.

\section{Case Report}

A 31-year-old homeless man with severe cachexia presented with abdominal pain for 2 hours prior to admission. During the preceding 3 years, he had several sexual contacts in addition to a history of IV drug addiction. He had previously been in good health. He was unaware of any family history of immunodeficiency disorder or infectious disease. He had a history of night sweats and a $12 \mathrm{~kg}$ weight loss in a period of three months. For the preceding 3 weeks, he had suffered from mild dyspnea. He was afebrile (Temperature: $36.5^{\circ} \mathrm{C}$ ) and his pulse and respiratory rates were 88 and 26 per minute, respectively.

\section{Physical examination and para-clinical data}

A physical examination showed diffuse abdominal tenderness. Neither peripheral lymphadenopathy nor hepatosplenomegaly was detected. Auscultation of the heart revealed normal sounds. Diffuse rales were heard in a chest examination. An examination of the oral cavity, pharynx, larynx, salivary glands and skin was unremarkable. A neurological examination showed bilateral normal ankle-tendon reflexes, and sensory and motor exams were normal.

A chest X-ray showed a diffuse miliary pattern. Sonography revealed several para-aortic lesions of varying size, which were consistent with lymphoma. Laboratory data showed leukopenia [white blood cells: 1200/mL, lymphocytes: 26\%; polymorphonuclear leukocytes (PMN): 68\%], normocytic anemia [Hemoglubin (Hb): $115 \mathrm{~g} / \mathrm{L}$ ], first hour erythrocyte sedimentation rate (ESR) was $76 \mathrm{~mm} / \mathrm{h}$, C-reactive protein (CRP) was 40 $\mathrm{mg} / \mathrm{L}$ (normal, $<5 \mathrm{mg} / \mathrm{L}$ ), lactate dehydrogenase (LDH) and alkaline phosphatase were not high (400 IU/L; and $250 \mathrm{IU} / \mathrm{L}$, respectively), and alanine amino-transferase (ALT) and aspartate amino-transferase (AST) levels were in the normal range (45 and $40 \mathrm{IU} / \mathrm{L}$, respectively). Blood cultures remained sterile. Several other workups were all unremarkable.

\section{Outcome}

The patient continued to have abdominal pain and tenderness after admission, while no source of acute abdominal pain was identified. Diagnostic laparotomy was performed for the multiple para-aortic lymph nodes and they were removed for histopathological examination. Unfortunately, the patient developed respiratory arrest and died in the operating room. The cadav-
Correspondence: Marzieh Hosseini, Legal Medicine Research Center, Legal Medicine Organization, Tehran, Iran.

Tel/Fax: 6324100616

E-mail: m.hosseini3078@yahoo.com

Key words: coinfection, cytomegalovirus, Mycobacterium avium Complex, immunodeficiency virus.

Contributions: AHe, conceived the study, and participated in its design and coordination, and helped draft the manuscript; $\mathrm{MH}$ participated in the autopsy, histopathological diagnosis, writing of the report and revising it after submission. AHo participated in writing the report as well as critical revision of the manuscript.

Conflict of interests: the authors declare no potential conflict of interests.

Acknowledgements: this work was supported by the Legal Medicine Organization of Fars. The authors declare that they have no conflict of interest.

Received for publication: 16 September 2012. Revision received: 10 November 2012.

Accepted for publication: 12 November 2012.

This work is licensed under a Creative Commons Attribution NonCommercial 3.0 License (CC BYNC 3.0).

(C) Copyright A. Hedjazi et al., 2013

Licensee PAGEPress, Italy

Infectious Disease Reports 2013; 5:e2

doi:10.4081/idr.2013.e2

er was shifted to Legal Medicine Organization of Fars and an autopsy was performed 24 hours after death to determine the exact cause of death.

\section{Autopsy findings}

Multiple para-aortic lymphadenopathy was identified, and the diameter of the largest lymph node was $25 \mathrm{~mm}$. Gross examination of the lungs showed diffuse congestion and patches of consolidation. The liver and spleen were grossly unremarkable with a normal size. The central nervous system, heart, gastrointestinal tract, kidneys, bladder and all other organs showed no significant changes. Microscopic examination of the lungs showed a predominantly mononuclear inflammatory infiltrate in conjunction with edema and hyperplasia of the alveolar epithelium. A characteristic intra-nuclear CMV inclusion body was detected (Figure 1). The immunoperoxidase technique using monoclonal antibody (Clone DDG9 + CCH2, Dako, Denmark) confirmed the diagnosis of CMV infection. Examination of the enlarged lymph nodes showed diffuse replacement of nodal architecture by foamy, large and plump macrophages. 
The cytoplasm of these macrophages contained large amounts of a diastase resistant Periodic Acid Schiff (PAS) positive material due to the presence of bacilliform bodies. Acid fast stain showed macrophages containing innumerable organisms (Figure 2). CMV intra-nuclear inclusions were also detected in these lymph nodes (Figure 3). No fungi or bacteria were identified with Gram, PAS, metenamin silver or WarthinStarry stains. Serological studies for HIV antibody on peripheral blood (preserved in the hospital blood bank) were positive for enzyme immunoassay, which confirmed a western blot assay. Serological tests for hepatitis A, B, C and syphilis showed negative results. Examination

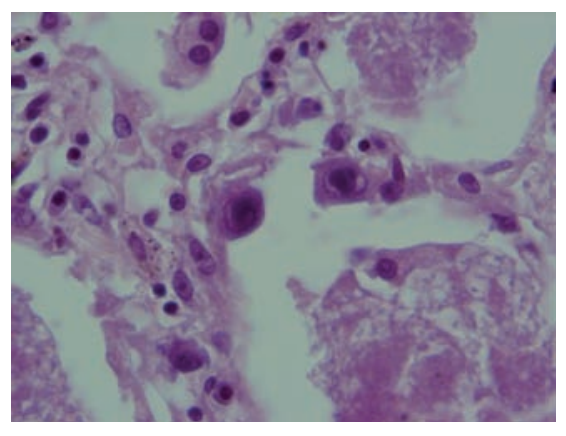

Figure 1. Lung, intranuclear cytomegalovirus inclusion body (Hematoxylin $\&$ Eosin $\times 400)$.

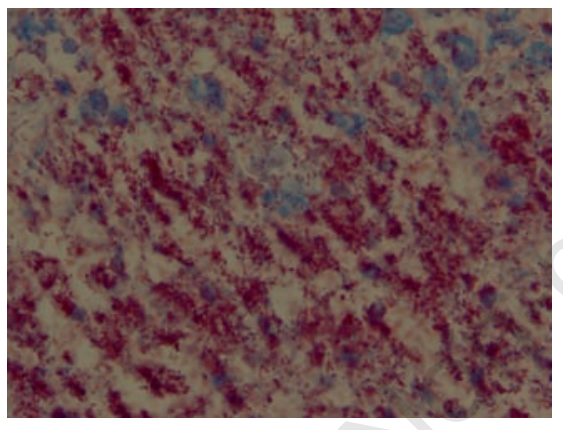

Figure 2. Lymph node, acid fast positive bacilli (Hematoxylin \&Eosin $\times 400)$.

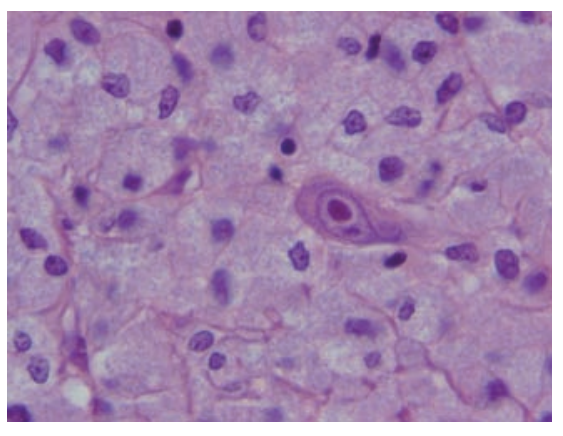

Figure 3. Lymph node, intranuclear cytomegalovirus inclusion body and plump histiocytes replacing the nodal architecture (Hematoxylin \& Eosin $\times 400$ ). of other organs showed no specific changes, either grossly or microscopically.

\section{Discussion and Conclusions}

We report here the discovery of 3 infections or coinfections at the same time in a young male patient who was hospitalized for abdominal pain. Although ongoing medical progress has allowed a large number of patients with HIV infection to survive despite profound immunosuppression, multiple unusual pathogens in AIDS patients can be complex. ${ }^{8}$ Recurrent and polymicrobial infections are frequently observed in HIV-infected patients, but coinfection is rarely reported. In a study on various opportunistic agents in HIV-positive persons, co-infection by Cryptococcus neoformans together with Mycobacterium aviumintracellulare was found when a CD4-positive lymphocyte count was as low as $3-20 / \mu \mathrm{L} .{ }^{9}$ Edmonson et al. reported histopathological and clinical findings of bacillary angiomatosis involving the palpebral conjunctiva with concomitant infection by CMV and mycobacterium species in a patient with AIDS. ${ }^{10}$ Rovery et al. reported an AIDS patient with a primary clinical presentation suggestive of bacillary angiomatosis. On clinical work-up, MAC and CMV were found in cutaneous lesions. ${ }^{8}$ Bernit et al. described Bartonella quintana and Mycobacterium tuberculosis coinfection in an HIV-infected patient with lymphadenitis. ${ }^{11,12}$ CMV and MAC were isolated from HIV patients and an association of CMV and atypical mycobacteria was found in cutaneous lesions of AIDS patients, ${ }^{7}$ but concurrent lymph node involvement of CMV and MAC has not been reported yet. To the best of our knowledge, this is the first report of simultaneous lymph node coinfection of CMV and MAC in AIDS and an unusual presentation of this coinfection as acute abdomen. It is important to consider the possibility of complex infections in immunocompromised individuals and to search for multiple agents in biopsy specimens in such patients.

HIV typically enters through mucosal epithelia. The subsequent pathogenetic events and clinical manifestations of this infection can be divided into three phases: i) an acute retroviral syndrome, ii) a middle chronic phase and, iii) AIDS. Acute retroviral syndrome develops in 40$60 \%$ of individuals who acquire a primary infection. This phase typically occurs 3 to 6 weeks after infection and clinically is associated with nonspecific symptoms resembling a flue like syndrome. During chronic phase few or no clinical manifestations of the HIV infection are present. After a variable period serious opportunistic infections, secondary neoplasms or clinical neurologic disease emerge and the patient is said to have developed AIDS. ${ }^{9}$ This disease can be diagnosed in every these phases. This case report is the first manifestation of AIDS in this patient.

\section{References}

1. Ananda S, Shaohua Z, Fan Y, Liang L. HIVnegative drug addict diagnosed with AIDS and tuberculosis at autopsy: a case report and brief review of literature. J Forensic Leg Med 2011;18:136-8.

2. Lazenby GB. Trichomonas vaginalis screening and prevention in order to impact the HIV pandemic: Isn't it time we take this infection seriously? Infect Dis Rep 2011;3:e4.

3. Jain SB, Wig N, Nagpal SJ, et al. Evaluation of the current management protocols for prophylaxis against Pneumocystis jiroveci pneumonia and other opportunistic infections in patients living with HIV/AIDS. AIDS Care 2011; 23:846-50.

4. Aberg JA, Yajko DM, Jacobson MA. Eradication of AIDS-related disseminated mycobacterium avium complex infection after 12 months of antimycobacterial therapy combined with highly active antiretroviral therapy. J Infect Dis 1998;178:1446-9.

5. Corti M, Palmero D. Mycobacterium avium complex infection in HIV/AIDS patients. Expert Rev Anti Infect Ther 2008;6:351-63.

6. Rosai J. Lung and pleura. In: Rosai and Ackerman's surgical pathology. 10th ed. Mosby; 2011. p. 361.

7. Núñez M, Miralles ES, Hilara Y, et al. Concurrent cytomegalovirus, M. tuberculosis and M. avium-intracellulare cutaneous infection in an HIV patient. J Dermatol 1997;24:401-4.

8. Rovery C, Rolain JM, Lepidi $\mathrm{H}$, et al. Bartonella quintana coinfection with Mycobacterium avium complex and CMV in an AIDS patient: case presentation. BMC Infect Dis 2006;6:89.

9. Arastéh K, Cordes C, Futh U, et al. Co-infection by Cryptococcus neoformans and Mycobacterium avium intracellulare in AIDS. Clinical and epidemiological aspects. Mycopathologia 1997-1998;140: 115-20.

10. Edmonson BC, Morris WR, Osborn FD. Bacillary Angiomatosis with Cytomegaloviral and Mycobacterial Infections of the Palpebral Conjunctiva in a Patient with AIDS. Ophthal Plast Reconstr Surg 2004;20:168-70.

11. Bernit E, Veit V, La Scola B, et al. Bartonella quintana and Mycobacterium tuberculosis coinfection in an HIV-infected patient with lymphadenitis. J Infect 2003;46:244-6.

12. Diseases of the immune system. In: Kumar V, Abbas AK, Fausto N, Aster J. Robbins and Cotran Pathologic Basis of Disease. 8th ed. Hoboken: Saunders; 2010. pp. 150-1. 